\title{
Short-Term Throughput Maximization for Battery Limited Energy Harvesting Nodes
}

\author{
Kaya Tutuncuoglu \\ Aylin Yener \\ Wireless Communications and Networking Laboratory (WCAN) \\ Electrical Engineering Department \\ The Pennsylvania State University, University Park, PA 16802 \\ kxt234@psu.edu_yener@ee.psu.edu
}

\begin{abstract}
Deploying energy harvesting nodes can significantly extend the lifetime of a battery powered wireless network. On the other hand, such systems call for specific design principles to efficiently utilize the dynamic levels of instantaneously available energy. In contrast to the conventional wireless networks, transmission policies for these systems should take into account the battery recharge process of the node as well as its battery capacity. In this work, we focus on the problem of finding the optimal transmission policy to maximize the short-term throughput of an energy harvesting transmitter node with power control and a limited energy storage capacity. The transmission policy in this problem is strictly constrained with both the energy recharge process and the energy storage capacity of the node. A discrete model with packets of energy arrivals is considered for energy recharge. Under the assumption of an increasing concave power-rate relationship, the short-term throughput maximizing transmission policy is identified in this paper. The necessary conditions such a policy should satisfy are identified and an algorithm to yield the unique policy that satisfies these conditions is presented. The performance of this algorithm is then observed through numerical results.
\end{abstract}

\section{INTRODUCTION}

Recently with the ubiquitous use of wireless devices powered with batteries, improving the efficiency and performance of such wireless networks is attracting more attention [1]. Solely battery powered systems suffer from a limited lifetime, whereas rechargeable networks with energy alternatives such as energy harvesting can function indefinitely [2]. The performance of these systems depends on efficient utilization of available energy and the energy to be harvested. Towards assessing the fundamental performance limits of energy harvesting systems, in this work, we consider the simplest such setting and find optimal transmission policies for short-term throughput maximization of a finite battery energy harvesting wireless node.

Energy efficient communications for systems with nonrechargeable battery powered nodes have been studied extensively [3]-[7]. In [3], energy efficient communications under a deadline constraint is considered, resulting in a minimum energy packet scheduling algorithm. In [4], the problem with individual deadline or buffer constrained systems with data arrivals is solved.

The interest in energy harvesting nodes in wireless networks has grown more recently [8]-[10]. A discrete-time battery

This work was supported by NSF Grant CNS 09-64364. model for the problem of energy aware routing in wireless networks powered with renewable energy was introduced in [8]. In [9], a queue stabilizing transmission policy is developed for a recharging battery powered transmitter. This is a modified adaptive backpressure policy that is shown to be asymptotically optimal for sufficiently large battery capacity. The reference that is most relevant to this work is [10], where transmission policies that minimize the transmission completion time of a given amount of data were found for an energy harvesting node under the assumption of an infinite capacity battery.

In this paper, we consider the problem of maximizing the transferred data in an energy harvesting node under a deadline constraint, i.e. the short-term throughput. Aside from the problem definition itself, this work differs from reference [10] in that we consider the case that the harvested energy can be stored up to an energy level that we shall call the battery capacity. Energy beyond this level cannot be stored and would be lost. This constraint models practical batteries and extenuates the necessity for energy-wise transmission policies. Under this setting, we solve for the optimum transmission policy and present an algorithm that produces the optimal solution. Numerical results suggest that employing optimum power allocation is particularly beneficial for transmitters with limited battery capacity or high variance energy harvests.

\section{System Model and Problem Definition}

We assume a single link continuous time system and a transmitting node with power control. Specifically, the transmitter can transmit with any desired finite power $P(t)$, achieving a corresponding instantaneous rate $r(p(t))$ at time $t$. Here $r($. is a non-negative, increasing, strictly concave function that we will refer to as the power-rate function in the sequel. Using a power-rate function of this form is fairly common [4], [10], and is clearly valid for the AWGN channel.

We consider a finite-capacity battery system that recharges its energy through harvesting. The battery can hold up to energy $E_{\max }$ with the overflowing energy being lost. It is assumed that there is no battery wear or fatigue, i.e., the energy storage capacity remains constant throughout the lifetime of the network. We define energy-feasibility as the property of keeping the battery energy below its capacity and nonnegative, i.e. within the interval $\left[0, E_{\max }\right]$. 


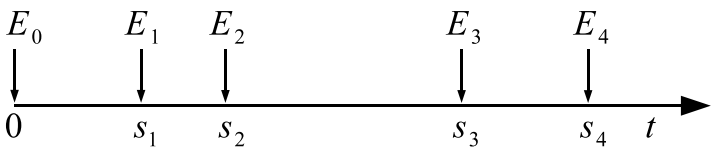

Fig. 1: Energy harvesting model.

The energy recharge process is modeled as a discrete process with packets of energy harvests arriving at specific time instances, all of which is known by the transmitter before start of transmission. Thus, the problem could be identified as the offline optimization problem. As shown in Figure 1, the $n^{\text {th }}$ energy harvest arrives at time $t=s_{n}$ and has an energy content of $E_{n}$, where $\left\{E_{n}, s_{n} \in \mathbb{R}^{+}\right\}$. The $0^{\text {th }}$ energy arrival is assumed to be at time $t=0$, corresponding to the initial battery state. The limited battery constraint acts when the harvested energy $E_{n}$ exceeds the available space in the battery at time $s_{n}$, at which point the battery is charged to its capacity and the remainder of the energy is discarded. The discarded energy is lost irreversibly as its consumption without storage would require infinite instantaneous power, which goes against the realistic system definition.

This model enforces two sets of constraints on the amount of energy available at any time instant, These arise either because a sufficient amount is not yet harvested, or can not be stored in the battery. Thus, we have two sets of energyfeasibility constraints on the power allocation function the transmission policy yields. Any energy-feasible power allocation $p(t)$ ensures that the battery state stays within $\left[0, E_{\max }\right]$ at every instant of transmission. Note that $p(t)$ being nonnegative implies that battery state reaches its local maxima and minima at arrival instances, and thus it is sufficient to check the feasibility conditions at the boundaries $s_{n}$ for each $n$. We define the set of energy-feasible power allocation functions, $\mathfrak{P}$, as:

$$
\begin{gathered}
\mathfrak{P}=\left\{p(t) \mid 0 \leq \sum_{k=0}^{n-1} E_{k}-\int_{0}^{t^{\prime}} p(t) \mathrm{dt} \leq E_{\text {max }},\right. \\
\left.\forall n>0, s_{n-1} \leq t^{\prime}<s_{n}\right\}
\end{gathered}
$$

where the former inequality ensures that enough energy is harvested so far, i.e. energy causality, and the latter inequality ensures that battery capacity is not exceeded. Power allocations allowing battery overflows are physically possible, but are not included in the energy-feasible set $\mathfrak{P}$ due to being strictly suboptimal as stated in subsequent sections of this paper. The convexity of set $\mathfrak{P}$ can easily be verified by employing the distributive property of integral over any convex combination of two feasible power functions.

The goal of the short-term throughput maximization problem is to find the optimum power allocation that maximizes the total data departed from an energy harvesting node within the deadline constraint. The constraints of this problem are the feasibility set $\mathfrak{P}$ and the deadline $T$ after which transmission is useless. The goal is to maximize the total number of bits departed in the time interval $[0, T]$ over $p(t)$. The problem can be expressed as:

$$
\max _{p(t)} \quad \int_{0}^{T} r(p(t)) \mathrm{dt}, \quad \text { s.t. } \quad p(t) \in \mathfrak{P}
$$

where $r(p(t))$ is the power-rate function. Next, the solution to this problem is sought.

\section{Short-Term Throughput MAXimization}

This section considers the short-term throughput maximization problem for an energy harvesting node with a deadline constraint. First, the necessary properties of the optimal policy are presented. Then, an algorithm is introduced to generate the policy that satisfies all the necessary conditions and the optimality of this policy is shown.

\section{A. Optimality conditions}

The following properties point out necessary conditions for a power allocation policy to be optimal. These conditions provide valuable insight into the development of the algorithm proposed in Subsection III-B and the proof of its optimality. These properties are presented in detail as Lemmas and proved in [11].

Property 1: The optimal power allocation policy dictates the transmission power/rate remains constant between energy arrivals. The power/rate can change only when a new energy packet arrives.

Property 2: When the optimal power allocation policy is employed, the battery never overflows, i.e., the available energy never exceeds battery capacity $E_{\max }$, unless it is completely depleted.

Remark 1: Energy packets larger than the capacity of the battery $E_{\max }$ can be truncated to the battery capacity, knowing that the rest of the energy is bound to be lost. Such an operation would generalize Property 2 to all battery states, as the case of empty battery overflow is eliminated with the truncation. We will assume this truncation in the sequel.

Property 3: When the optimal power allocation is employed, the transmission power/rate cannot change unless the battery is either full or completely depleted.

Property 4: For the optimal power allocation, transmission power/rate can increase only when the battery is depleted. Similarly, it can decrease only when the battery is full.

Property 5: Using optimal power allocation, we expend all energy by the end of the transmission.

\section{B. Throughput Maximizing Policy}

We begin by presenting the definitions of some variables used in the policy. Properties 1-5 in Section III-A indicate that the power allocation policy will consist of epochs of constant power, with epochs starting and terminating only at energy arrival instances. We denote the time instance at which the $n^{t h}$ such interval ends by $i_{n}$, and the power allocated to the epoch $\left[i_{n-1}, i_{n}\right]$ by $p_{n}$. Due to Property 1 , the epoch boundaries $i_{n}$ are known to fall on the set of energy arrivals $s_{m}$. Hence, the optimal power allocation takes the following form:

$$
p(t)= \begin{cases}p_{n} & i_{n-1}<t<i_{n}, i_{0}=0 \\ 0 & t>i_{n_{\max }}\end{cases}
$$


Remark 2: Once the specifics of the first epoch $t=\left[0, i_{1}\right]$ is determined, the remainder of the problem can be considered as a separate throughput maximization problem. That is, given the duration of the first epoch, $\overline{i_{1}}$, and the amount of information sent in this epoch, $\overline{i_{1}} \cdot r\left(\overline{p_{1}}\right)$, it remains to solve for the optimal power allocation for the modified problem with energy arrival times shifted by $\overline{i_{1}}$, a new initial battery state $E_{0}+E_{1}-\overline{i_{1}} \cdot \overline{\overline{p_{1}}}$, and a new deadline constraint of $T-\overline{i_{1}}$. This means that, once the first epoch of the optimal allocation is identified, the remaining epochs and the corresponding power levels can be recursively with the same algorithm, using updated states and shifted arrival times. Thus, we shall focus on determining the initial epoch and the power level in it. The modified optimization problem to find the next epoch will be referred to as the shifted optimization problem.

We define two sets of transmission powers $\left\{p_{0}[1], p_{0}[2], \ldots\right\}$ and $\left\{p_{\max }[1], p_{\max }[2], \ldots\right\}$, where $p_{0}[n]$ and $p_{\max }[n]$ are the constant power levels during $\left[0, s_{n}\right]$ that would result in an empty battery at $s_{n}^{-}$or a full battery at $s_{n}^{+}$respectively. We then define the set $\mathbf{P}=\{\mathbf{P}[1], \mathbf{P}[2], \ldots\}$ with elements $\mathbf{P}[n]$ as the closed intervals between the corresponding elements of the two sets, as defined in Equation 6. This translates to a range of constant power levels for the $n^{t h}$ arrival that is energy-feasible at $s_{n}$ when the feasibility at previous arrivals are disregarded. Thus, we have

$$
\begin{aligned}
p_{0}[n] & =\frac{\sum_{k=0}^{n-1} E_{k}}{s_{n}}, & s_{n} & <T \\
p_{\max }[n] & =\max \left\{\frac{\sum_{k=0}^{n} E_{k}-E_{\max }}{s_{n}}, 0\right\}, & & s_{n}<T \\
\mathbf{P}[n] & =\left[p_{\max }[n], p_{0}[n]\right], & s_{n} & <T \\
\mathbf{P}\left[n_{\max }\right] & =\left[p_{0}\left[n_{\max }\right]\right], & s_{n_{\text {max }}} & =T
\end{aligned}
$$

Here, (7) creates a virtual arrival point $s_{n_{\max }}$ at the deadline $T$, and assigns to the feasible power range $\mathbf{P}\left[n_{\max }\right]$ the single power level depleting the battery at $T$, thus satisfying Property 5. Based on this definition of the feasible power range, it can easily be deduced that for the first constant power step starting from $t=0$ to extend to the $i^{t h}$ energy arrival without violating energy-feasibility, its power level should be contained in the range $\mathbf{P}[n]$ for all previous $n=1, \ldots, i$, i.e., the step should be feasible through all arrivals it extends over. This yields an upper bound $n_{u b}$ on the index of the first step, that can be calculated as

$$
n_{u b}=\max \left\{n \mid \bigcap_{k=1}^{n} \mathbf{P}[k] \neq \varnothing, n=1,2, . ., n_{\max }\right\}
$$

as none of the later arrivals have a candidate constant power level contained in the feasible power ranges $\mathbf{P}[n]$ of the previous arrivals. This gives us a range of feasible power levels and the duration of the first epoch.

A graphical depiction of these quantities is provided in Figure 2. It shows the feasible energy tunnel of the energy harvesting transmitter. The upper wall represents the cumulative energy harvested prior to time $t$, thus providing an upper bound to the total transmission energy spent up to $t$. Similarly, the lower wall is the upper wall shifted down by an amount of $E_{\max }$, providing a lower bound below

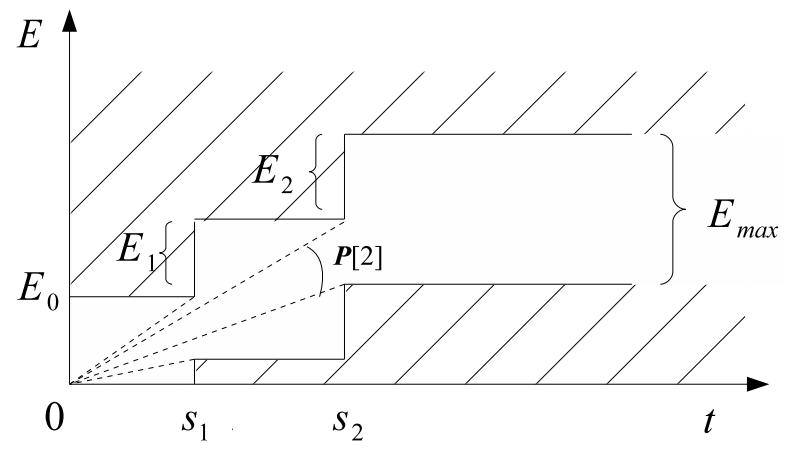

Fig. 2: The feasible energy tunnel.

which the unused energy amount exceeds battery capacity. The cumulative energy spent by the power allocation policy forms a continuous line within this tunnel, the slope of which gives the transmission power. The set of energy-feasible power allocations $\mathfrak{P}$ must therefore have integrals lying within this tunnel. Note that this graphical approach is similar to the tunnel for cumulative data transferred in [4], whereas here we have an energy tunnel. In this figure, the sets $p_{0}[n]$ and $p_{\max }[n]$ correspond to the slopes of lines from the origin to each of the corner points in the tunnel as shown with dashed lines. The range $\mathbf{P}[n]$ represents the slope set of lines passing through the $n^{\text {th }}$ opening in the tunnel, and is marked with an arc on the figure for $n=2$.

The analysis presented so far aims at insights on the optimal power allocation. Based on these, the following algorithm determines the throughput maximizing power allocation for a transmitter node with initial energy $E_{0}$, energy arrivals $E_{n}$ at times $s_{n}$, battery capacity $E_{\max }$ and deadline constraint $T$ : Throughput Maximizing Algorithm

1) Find the upper bound on the length of the step as $i_{u b}=$ $s_{n_{u b}}$ using (8). If $n_{u b}=n_{\max }$, transmit with constant power $\left(\sum_{k=0}^{n_{\max }} E_{k}\right) / T$ until the end of transmission.

2) Determine whether the next feasible power interval $\mathbf{P}\left[i_{\max }+1\right]$ falls below or above $\bigcap_{k=0}^{i_{\max }} \mathbf{P}[k]$. This determines the tendency of the policy to increase or decrease power.

3) If power tends to increase, set the first step as

$$
\begin{aligned}
& n_{1}=\max \left\{n \mid p_{0}[n] \in \bigcap_{k=0}^{n} \mathbf{P}[k]\right\}, \\
& i_{1}=s_{n_{1}}, \quad p_{1}=p_{0}\left[i_{1}\right]
\end{aligned}
$$

If power tends to decrease, assign

$$
\begin{aligned}
& n_{1}=\max \left\{n \mid p_{\max }[n] \in \bigcap_{k=0}^{n} \mathbf{P}[k]\right\}, \\
& i_{1}=s_{n_{1}}, \quad p_{1}=p_{\max }\left[i_{1}\right]
\end{aligned}
$$

4) Repeat algorithm for the shifted problem with updated parameters

$$
\begin{aligned}
E_{0}^{\prime} & =E_{0}+E_{1}-i_{1} \cdot p_{1}, \quad T^{\prime}=T-i_{1}, \\
n_{\max }^{\prime} & =n_{\max }-n_{1}, \quad E_{n}^{\prime}=E_{n+n_{1}}, \\
s_{n}^{\prime} & =s_{n+n_{1}}-i_{1}, \quad n=0, \ldots, n_{\max }^{\prime}
\end{aligned}
$$


For a verbal description of the algorithm, consider the feasible energy tunnel in Figure 2. The first step of the algorithm determines the longest constant power transmission that stays within this tunnel. The second step determines whether the most distant point on a wall you can reach from the origin is an upper bound or a lower bound. This is accomplished by comparing the first unreachable opening with the last reachable one. Finally, the third step selects the longest feasible constant transmission that ends in one of the sets $p_{0}[n]$ and $p_{\max }[n]$, allowing a change in transmission power for the rest of the problem.

Theorem 1: The proposed throughput maximizing algorithm yields the optimal power allocation policy.

Proof: Proof is by contradiction. We begin by assuming that there exists an optimal power allocation $\left\{\overline{p_{n}}\right\},\left\{\overline{i_{n}}\right\}$ such that $p_{1} \neq \overline{p_{1}}$ or $i_{1} \neq \overline{i_{1}}$. There are four possible distinct cases over which we will show contradictions on optimality, labeled in Figure 3 as I-IV. The main point behind the contradictions is the fact that a constant power transmission, when feasible, performs strictly better than any other alternative between two points within the feasible energy tunnel tunnel.

Figure 3(a) shows a case where the algorithm chooses to transmit with power $p_{\max }\left[n_{1}\right]$ for a duration $s_{n_{1}}$. This selection is based on point A lying on the upper tunnel boundary as implied by the second step of the algorithm. A power allocation policy can differ from this selection by either trespassing to the upper region I or the lower region II. Recall that a feasible power allocation forms a continuous line that stays within the tunnel throughout the transmission time. A power allocation extending to region II must then cross the algorithm's line at least once again before $s_{n_{1}}$. At this crossing point, the algorithm's transmission departs strictly more bits than the alternative, rendering any allocation extending to region II suboptimal. On the other hand, any policy extending to region I must cross the dashed line at some point to extend to the deadline, where a constant power transmission up to this crossing point is feasible and performs strictly better. Therefore, any allocation extending to region I cannot be optimal.

A parallel statement holds for the second case shown in Figure 3(b) where the algorithm chooses to transmit with power $p_{0}\left[n_{1}\right]$ instead. Similarly, due to the second step of algorithm, point B is known to lie on a lower boundary. A policy extending to region III has to cross the algorithm's line at some point in order to complete a feasible path to the deadline. At this crossing point it proves itself suboptimal as the algorithm's transmission is strictly better. On the other hand a policy extending to region IV has to cross the dashed line, at which point a constant power transmission up to that point is both feasible and better. Therefore no other policy can perform better than the constant power transmission shown with the solid line.

The termination step follows an analogous logic. Due to Property 5, it is known that the policy must terminate with a completely depleted battery at deadline $\mathrm{T}$, the point which the algorithm points to. The termination step suggests a constant power transmission to this point whenever feasible. As the constant power transmission is the optimal solution

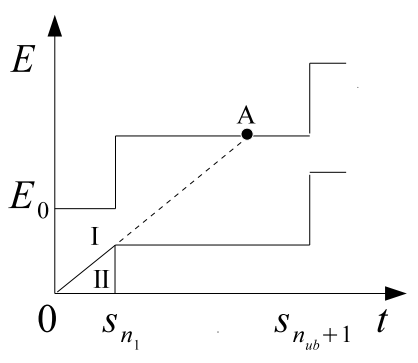

(a) $E_{\max }$ case

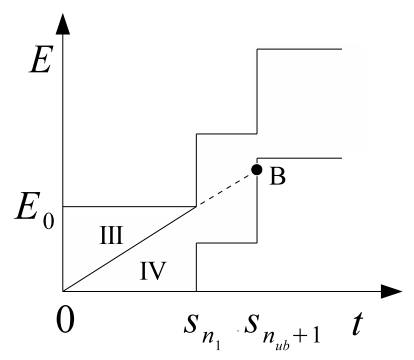

(b) $E_{0}$ case
Fig. 3: The optimal first step and suboptimal regions

for the unconstrained problem as in Property 1, it emerges as the solution to the energy constrained problem as well, provided that it is feasible. Consequently any alternative power allocation departing from this constant power transmission would be suboptimal, as the two policies have to terminate at the same point at which the constant power transmission clearly departs more bits.

In summary, any transmission policy that differs from the one found by the algorithm and still is energy feasible must be suboptimal. Consequently, each of our proposed algorithm's steps has to yield the optimal policy. Additionally, the algorithm yielding a unique output at each step indicates that the optimal policy is in fact unique.

\section{Simulation Results}

In this section, we demonstrate the behavior and the performance of the algorithms with simulations. We start with a sample simulation run of the algorithm presented in Section III-B. The energy arrival scenario is deterministic with energy arrivals of $E_{n}=\{2,1,6,4,8,1\}$ units at times $s_{n}=$ $\{0,2,4,5,7,11\}$ respectively, as shown in Figure 4(a). The energy tunnel of this system for a battery capacity $E_{\max }=10$ units is drawn in Figure 4(b) along with the result of the throughput maximization algorithm for a deadline constraint $T=12$.

The first step of the algorithm calculates the sets $p_{0}[n]$ and $r_{\max }[n]$ and the corresponding power ranges $\mathbf{P}[n]$. These ranges are displayed in Figure 4(c) with intervals along the power axis, with the black dot corresponding to the final range $\mathbf{P}\left[n_{\max }\right]$ that consists of a single point. The upper bound on $n_{1}$ is determined to be $n_{u b}=3$ as the fourth interval $\mathbf{P}[4]$ falls outside $\mathbf{P}[1] \cap \mathbf{P}[2] \cap \mathbf{P}[3]$. Based on the position of $\mathbf{P}[4]$, the longest feasible transmission with power in $p_{0}[n]$ is picked, and the first step of the algorithm is decided as $p_{1}=p_{0}[2], i_{1}=s_{2}$. When new intervals for the shifted problem are calculated beyond $i_{1}$, an opposite position for $\mathbf{P}\left[n_{u b}+1\right]$ is observed and a transmission within $p_{\max }[n]$ is picked instead. Finally, for the next shifted problem, the constant power transmission is found to be feasible and the algorithm is terminated.

To evaluate the average long term performance of the algorithm, we simulate it for longer realizations. Assuming a battery capacity $E_{\max }=100$ units, we generate energy 


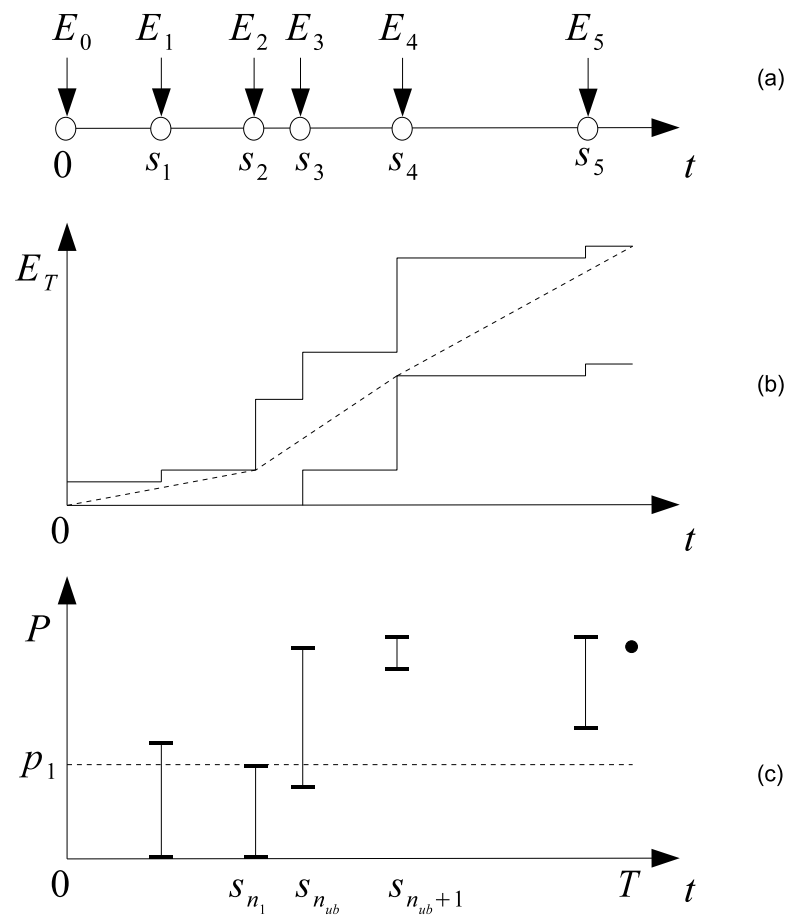

Fig. 4: Arrival scenario and results of the simulation run.

arrivals with a truncated gaussian distribution $\mathcal{N}(50,100)$ truncated to $\left[0, E_{\max }\right]$. The inter-arrival times $\left(s_{i}-s_{i-1}\right)$ are similarly generated with a gaussian distribution $\mathcal{N}(10,10)$ truncated to be strictly positive. for this case, Figure 5 compares our optimal power allocation algorithm for the throughput maximization problem with two alternative power allocations for a deadline of $T=10000 \mathrm{sec}$. First corresponds to transmission without regard to any battery or arrival constraints. This is the performance of a "traditional" transmitter with no energy harvesting, and is presented as an upper bound for our model where we are bound to conform to energy feasibility and battery constraints. Second is an alternative algorithmic approach named the on-off algorithm. It is based on the fact that for a strictly concave power-rate relationship, constant power transmission is the most efficient. The policy is such that, the transmitter operates with constant power when energy is available, and shuts off when energy is depleted. The constant power level is determined from the average energy arrival rate. As seen in Figure 5, the energy constraints of the problem result in a performance loss with respect to having perpetual energy. However, a major portion of this loss can be recovered with the incorporation of arrival information and use of variable power transmission. The optimal algorithm we present provides this remedy, and significantly outperforms the greedy on-off algorithm. Through further simulations involving various battery capacity and arrival statistics, we also observe that employing this algorithm is even more beneficial for systems with limited battery capacity or energy arrivals with large variations.

\section{CONCLUSION}

In this paper, we have solved the short-term throughput maximization problem for a battery limited energy harvesting

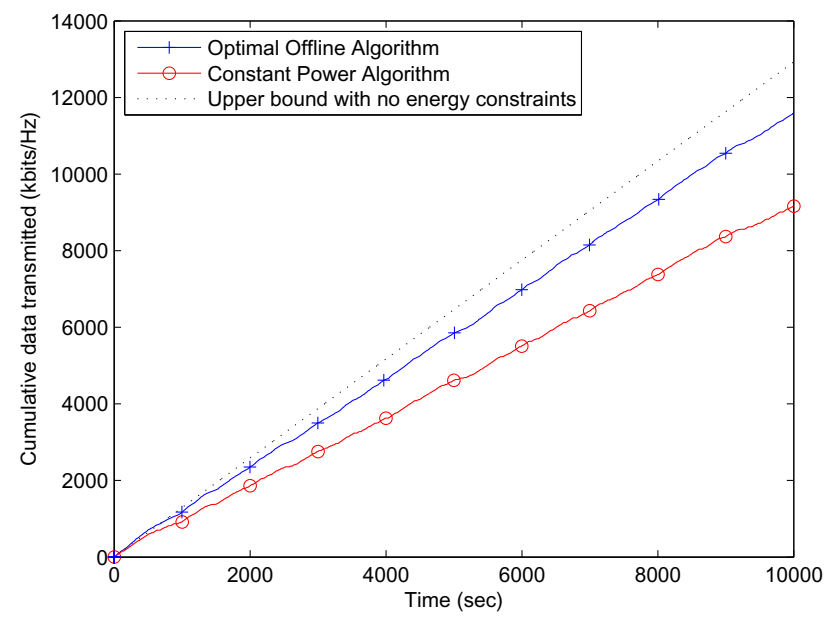

Fig. 5: Performance comparison of transmission policies.

transmitter node in a single link topology. We proposed an algorithm that yields the optimal solution of this problem and proved its optimality.

The findings of this paper provide insight to developing optimal transmission policies for nodes that have some notion of when and how much energy they can harvest. Future directions include developing online power allocation algorithms for causal systems or systems with limited or stochastic future energy harvests. There are also plenty of possible extensions for multiterminal system models from the single link model considered in this paper.

\section{REFERENCES}

[1] A. Ephremides, "Energy concerns in wireless networks," IEEE Transactions on Wireless Communications, vol. 9, no. 4, pp. 48-59, Aug. 2002.

[2] X. Jiang, J. Polastre, and D. Culler, "Perpetual environmentally powered sensor networks," in Proc. 4th International Symposium on Information Processing in Sensor Networks (IPSN), 2005, pp. 463-468.

[3] E. Uysal-Biyikoglu, B. Prabhakar, and A. El Gamal, "Energy-efficient packet transmission over a wireless link," IEEE/ACM Transactions on Networking, vol. 10, pp. 487-499, 2002.

[4] M. A. Zafer and E. Modiano, "A calculus approach to energy-efficient data transmission with quality-of-service constraints," IEEE/ACM Transactions on Networking, vol. 17, pp. 898-911, Mar. 2010.

[5] A. C. Fu, E. Modiano, and J. N. Tsitsiklis, "Optimal energy allocation and admission control for communications satellites," IEEE/ACM Transactions on Networking, vol. 11, no. 3, pp. 488-500, 2003.

[6] W. Heinzelman, A. Chandrakasan, and H. Balakrishnan, "An application-specific protocol architecture for wireless microsensor networks," IEEE Transactions on Wireless Communications, vol. 1, pp. 660-670, Oct. 2002.

[7] W. Ye, J. Heidemann, and D. Estrin, "An energy-efficient MAC protocol for wireless sensor networks," in IEEE INFOCOM, vol. 3, 2002, pp. $1567-1576$.

[8] L. Lin, N. Shroff, and R. Srikant, "Asymptotically optimal power-aware routing for multihop wireless networks with renewable energy sources," IEEE/ACM Transactions on Networking, vol. 15, pp. 1021-1034, Oct. 2007.

[9] M. Gatzianas, L. Georgiadis, and L. Tassiulas, "Control of wireless networks with rechargeable batteries," IEEE Transactions on Wireless Communications, vol. 9, pp. 581-593, Feb. 2010.

[10] J. Yang and S. Ulukus, "Transmission completion time minimization in an energy harvesting system," in 44th Annual Conference on Information Sciences and Systems, Princeton, Mar. 2010.

[11] K. Tutuncuoglu and A. Yener, "Optimum transmission policies for battery limited energy harvesting nodes," Submitted to IEEE Transactions on Wireless Communications, available at: http://arxiv.org/PS_cache/arxiv/pdf/1010/1010.6280v1.pdf. 